\title{
Manifestaciones orales del síndrome del cromosoma 18 en anillo
}

\section{Oral manifestations in chromosome 18 ring}

Rafael Celestino Souza ${ }^{1,2, a}$, Ruth Y. Andia-Merlin ${ }^{1, b}$, Carlos E. Allegretti ${ }^{1, c}$, Luiz F. Scabar $^{\text {ld }}$, Elcio M. Giovani ${ }^{1, e}$.

\section{RESUMEN}

El síndrome del cromosoma 18 en anillo es un desorden inusual cromosómico con varios cambios generalizados en el cuerpo y boca. Este artículo presenta el reporte del caso de un niño con síndrome del cromosoma 18 en anillo con retardo mental. Esta combinación raramente estudiada, presenta cambios generales y bucales predominantes y retención prolongada de dientes deciduos. El odontólogo debe estar preparado para detectar estos hallazgos en este tipo de pacientes y tratarlos precozmente proporcionando mejoras en su calidad de vida.

PALABRAS CLAVE: Cromosoma 18, retardo mental, terapia de comportamiento. (Fuente: DeCS BIREME)

\section{SUMMARY}

Ring chromosome 18 syndrome is a rare chromosomal disorder, with many changes in the general body and mouth. This article reports the case of a child with the syndrome ring chromosome 18 and mental retardation. This combination rarely studied shows oral and general predominant changes and prolonged retention of deciduous teeth. The dentist must be prepared to recognize these findings and treat them at early stages to promote improvements in their quality of life.

KEYWORDS: Chromosome 18, mental retardation, behavior therapy. (Source: MeSH NLM)

Facultad de Odontología, Universidad Paulista. São Paulo, Brasil.

Facultad de Odontología, Universidad de São Paulo. São Paulo, Brasil.

Estudiante de PhD en Odontopediatría. Profesor de Pacientes con Necesidades Especiales y Gestión Hospitalaria. rafacst@gmail.com

Maestría en Restauración y Profesora de la Clínica Integral y de Pacientes con Necesidades Especiales. ruthmerlin@hotmail.com

PhD en Clínica Integral. Director del Instituto de Ciencias de la Salud. allegretti@unip.br

PhD en Salud Pública. Profesor de Salud Pública y Vice Director del Instituto de Ciencias de la Salud. luizfelipe@unip.br

PhD en Clínica Integral. Docente de pre y posgrado de la Clínica Integral y Pacientes con Necesidades Especiales. elciomg@unip.br 


\section{INTRODUCCIÓN}

El síndrome del cromosoma 18 en anillo (MIM \# 601808) fue descrito por primera vez en la década de los 60's y desde entonces, han sido publicados en el mundo más de 80 reportes de caso según la Oficina de Enfermedades Inusuales del Instituto Nacional de Salud (Office of Rare Diseases of National Institutes of Health). Esto quiere decir que el cromosoma 18 en anillo o un subtipo del mismo, afecta menos de 200000 personas en la población norteamericana, considerándose un síndrome raro $(1,2)$.

Los mecanismos de formación de un cromosoma en anillo involucran generalmente deleciones de los extremos caudales de ambos brazos de un cromosoma y fusión de estos extremos libres fracturados. De esta manera, queda faltando una parte tanto del brazo largo como del brazo corto y, dependiendo del tamaño de la exclusión en cada extremidad del cromosoma, la apariencia fenotípica varía de normal a malformaciones graves. La incidencia y tipos de malformaciones congénitas son semejantes a aquellas que ejercen la deleción:18 (q21-qter), pero es posible que adquieran características tanto de $18 \mathrm{p}$ - como también de 18q-. Las técnicas de alta resolución citogenética molecular son útiles en la caracterización de los casos de mosaicismo y dinámica para establecer la relación entre la pérdida o ganancia de material cromosómico y el fenotipo. Algunos estudios relatan que no existe un fenotipo característico de esta condición, pues este va a depender de la localización y extensión del segmento eliminado $(1,3)$. El diagnóstico de este síndrome es realizado a través del examen de cariotipo completo de células amnióticas y/o del análisis citogenético del cordón umbilical (4).

Los pacientes que presentan cromosoma 18 en anillo comparten características clínicas del síndrome 18q-, tales como hipotonía, mala coordinación, microcefalia, anormalidades auditivas y malformaciones de los órganos genitales. Con menos frecuencia, pueden presentarse también ciertas características comunes con el síndrome $18 \mathrm{p}$, como una deficiencia en el crecimiento (leve a moderada) y ptosis (5).

Los individuos con síndrome del cromosoma 18 en anillo generalmente presentan holoprosencefalia, causada por falta de división del lóbulo frontal del cerebro del embrión e incapacidad de formar los hemisferios cerebrales bilaterales, que conlleva defectos en el desarrollo de la cara y en la estructura y funcionamiento del cerebro. En la mayoría de los casos se describen cambios en el desarrollo cerebral más lentos, donde se puede presentar un perjuicio del Coeficiente Intelectual $(\mathrm{CI})$ de leve a severo $(4,5)$. De la misma manera, se han identificado algunos problemas cardíacos, afectando el corazón en cerca de $30-40 \%$ de los niños y niñas con diferentes manifestaciones, siendo más comunes los defectos septales auriculares o ventriculares. La visión se ve también afectada, encontrándose normalmente estrabismo y movimientos involuntarios del globo ocular (1). Con relación a las alteraciones metabólicas, este tipo de pacientes frecuentemente sufre de obesidad, deficiencia de la hormona de crecimiento (HC), hipogonadismo hipogonadotrófico asociado, disfunción neurosecretora de $\mathrm{HC}$, diabetes mellitus tipo 1 e hipotiroidismo $(2,3,5)$.

Además de los hallazgos clínicos anteriormente mencionados, pueden estar presentes las siguientes condiciones: baja estatura, hipoplasia del tercio medio de la cara, boca de carpa, anti-hélices y antitragos prominentes, deformidades en los pies, manos pequeñas con dedos finos, hipotonía muscular, retardo motor, implantación posteriorizada y baja de las orejas y pliegues epicánticos $(1,4,6)$.

\section{Reporte del caso}

Siguiendo los preceptos éticos de investigación, este trabajo fue aprobado por un Comité de Ética en Investigación (CEP/UNIP n. 642/09) y sus tutores firmaron un Consentimiento Informado para participar de la investigación.

Paciente de género masculino de 10 años de edad que llegó al Centro de Estudios y Atención de Pacientes Especiales (CEAPE) refiriendo necesidad de tratamiento odontológico, portando clínicamente las características del síndrome y con historia clínica que confirmaba la patología del cromosoma 18 en anillo (Figura 1). Primogénito, de padres aparentemente normales y sin características de consanguinidad. El paciente refirió estar insatisfecho con la estética dentaria y con la demora en la exfoliación de los mismos. En la historia clínica médica se encontró que 
el paciente presentaba baja inmunidad, enfermedades pulmonares e historia pasada de alergia a la anestesia general (bajo estudio). El paciente no presentaba obesidad, ni alteraciones tiroideas; sin embargo, se observó un ligero retraso en su crecimiento, además de presentar manos pequeñas.

El abordaje para el tratamiento de este paciente se inició con técnicas de comportamiento y adaptación como "Decir-Mostrar-Hacer", refuerzos y control de voz. Estas técnicas probaron ser efectivas para este paciente, permitiendo todos los procedimientos clínicos.

Al examen clínico extraoral se encontraron las siguientes alteraciones faciales (Figura 1): pliegues epicánticos, hipertelorismo, tercio frontal y puentes supraciliares prominentes, fisuras palpebrales oblicuas, boca de carpa, labio superior fino, puente nasal deprimido y cuello corto. Al evaluar el perfil del paciente es posible observar mentón pequeño y baja implantación de las orejas (Figura 2). Al examen clínico intraoral, el hallazgo clínico más relevante de la oclusión fue la mordida abierta (Figura 3); y en el maxilar se observó: anodoncia de la pieza 52,



Figura 1. Vista extraoral de un paciente con síndrome del cromosoma 18 en anillo, mostrando: puente nasal plano, ojos almendrados, nariz respingada y punta nasal alta con labio superior más largo.

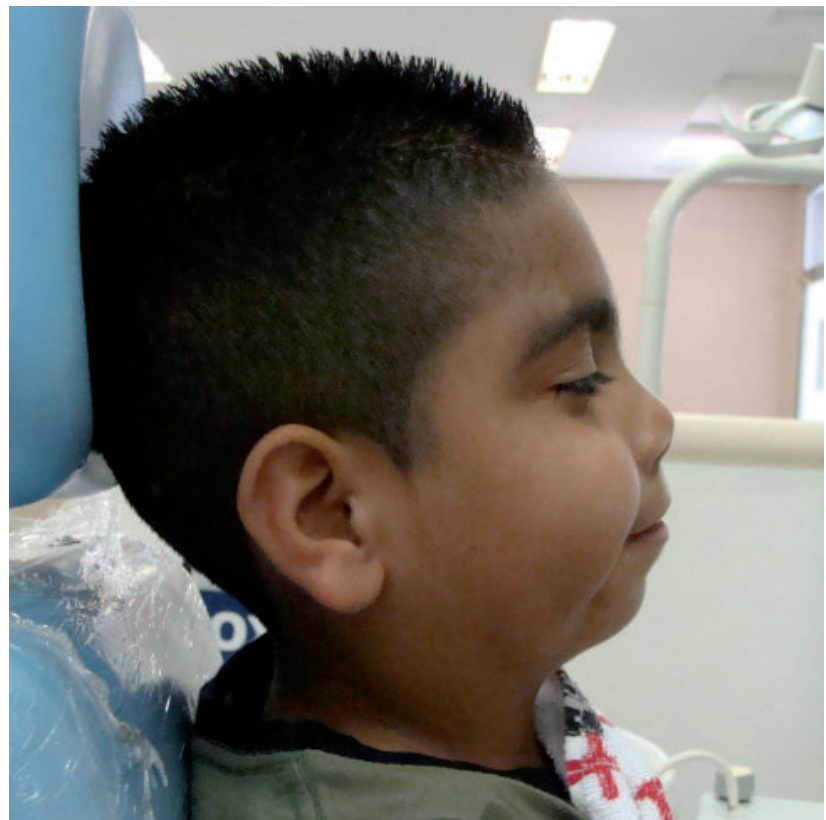

Figura 2. Perfil del paciente, enfatizando las orejas de implantación baja y subdesarrollo mandibular.

dientes cónicos, asimetría de la arcada superior, altos índices de placa ( $80 \%$ ) y caries dental (Figura 4). Los hallazgos en la mandíbula fueron: micrognatismo leve, hipoplasia del esmalte, retraso en la cronología de erupción, altos índices de placa y caries dental (Figura 5).

Las anomalías dentarias fueron detectadas a través del análisis de los modelos de estudio. Al examen radiográfico se observó que algunos dientes permanentes se encontraban en estadio de Nolla 9 y los deciduos correspondientes no habían exfoliado en el tiempo previsto. En la figura 5, se puede observar la pérdida o ausencia de matriz de esmalte en algunas piezas dentales: 74, 75 y 84 . No se confirmó ningún tipo de retraso en el desarrollo neuropsicomotor. Para el análisis de las características salivares se usó el test Dentobuff $\circledast$, que presentó resultados normales $(\mathrm{pH}$ 7,0; flujo salivar de $1,4 \mathrm{ml} / \mathrm{min}$ y capacidad tampón normal).

El tratamiento odontológico recomendado fue la adecuación del medio bucal, aplicación de barniz de flúor (Duraphat ${ }^{\circledR}$, Colgate), restauraciones con resina compuesta y extracción dental. Se realizó la extracción de las piezas 73, 74 y 84 (Figura 6). Durante la cirugía el paciente demostró ser cooperador y en el postoperatorio se observó una buena reparación del tejido, ausencia de edema y secreción. Actualmente, 


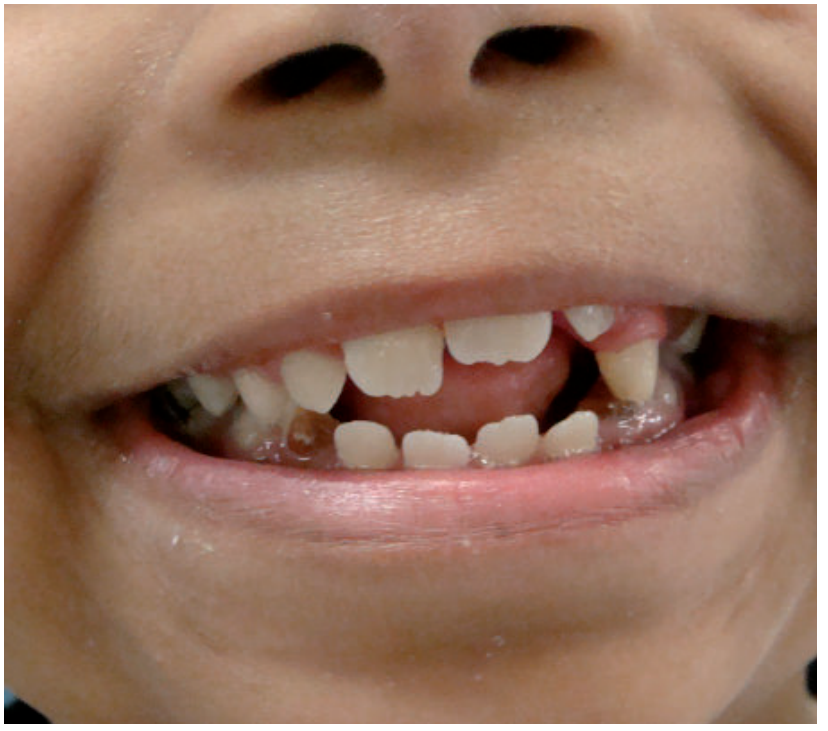

Figura 3. Aspecto bucal y sonrisa.

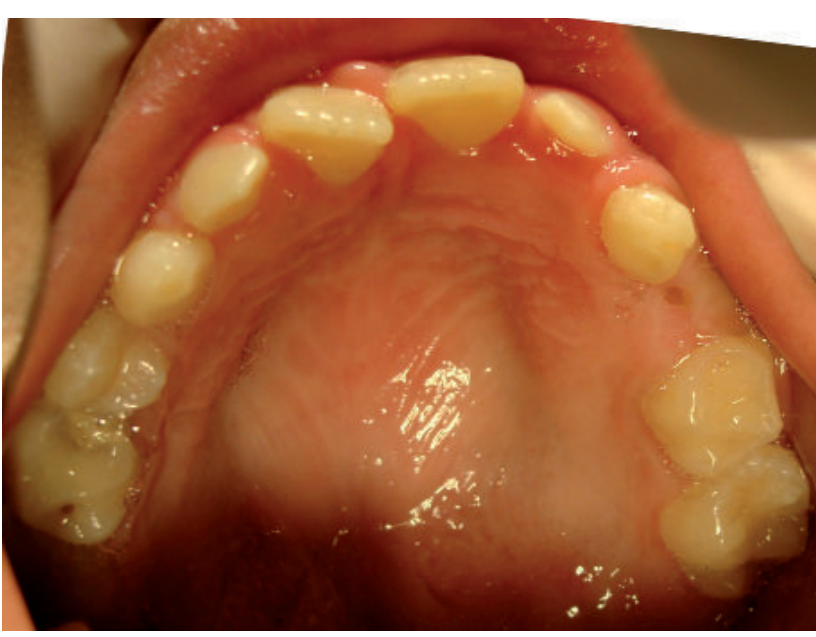

Figura 4. Dientes superiores y paladar.

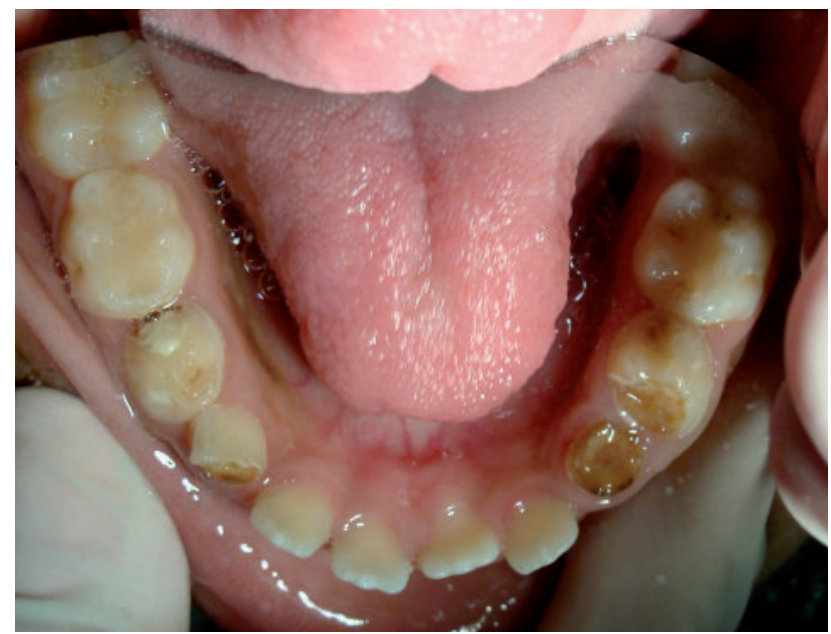

Figura 5. Mandíbula, caries dental e hipoplasia del esmalte.

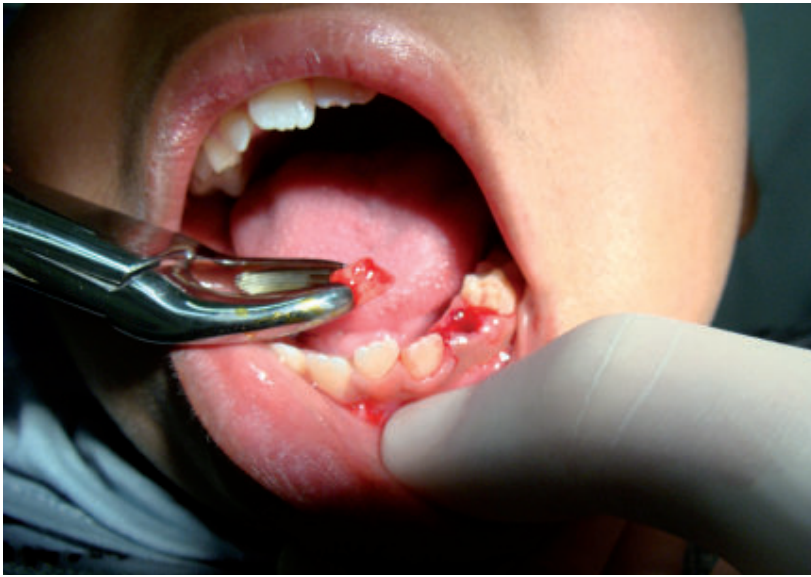

Figura 6. Procedimientos clínicos: extracción dental.

el paciente se encuentra en controles clínicos y radiográficos de los dientes con retención prolongada.

\section{DISCUSIÓN}

Las anomalías cromosómicas ocurren en $0,4 \%$ de los nacidos vivos. Los cromosomas en anillo han sido encontrados en los cromosomas humanos, y cuando éste sustituye un cromosoma normal, el resultado es la monosomía parcial. Sripanidkulchai y col. (7), al relatar un caso clínico de una paciente con cromosoma 18 en anillo, verificó que el fenotipo muchas veces se sobrepone a las alteraciones cromosómicas, por lo que no existe un consenso en las características de los pacientes. En el presente relato se describen las alteraciones clínicas generales y bucales de un niño con cromosoma 18 en anillo (46XY, R18), incluyendo alteraciones dismórficas, retraso del desarrollo cognitivo, hipotiroidismo e inmunoglobulina A deficiente.

Koç y col. (8) el año 2008, afirmaron en un estudio que las principales enfermedades neurológicas pueden acompañar raras anormalidades cromosómicas; como por ejemplo, el síndrome del cromosoma 18 en anillo. Al presentar un caso de esta rara condición, mostraron hallazgos de microcefalia, retardo mental, retraso de desarrollo, déficit de atención e hiperactividad, movimientos estereotipados, convulsiones y apariencia facial dismórfica. Concordando con nuestros hallazgos, donde estuvo presente la microcefalia, el retardo mental y apariencia facial dismórfica; contribuyendo a la identificación de la correlación genotipo-fenotipo en anomalías del cromosoma 18 (8). 
En el presente estudio el paciente presentó los siguientes hallazgos radiográficos: micrognatismo discreto, presencia de dientes permanentes en estadio de Nolla 9 y sus respectivos deciduos todavía presentes sin exfoliación y lesiones sugestivas de caries dental (Figuras 4 y 5). Con relación a las anomalías de oclusión, el paciente presentó mordida abierta y leve apiñamiento, un hallazgo clínico interesante y también encontrado por otros autores $(6,9,10)$.

Ono y col. (9) el año 2010, al comparar teleradiografías postero-anterior y lateral de cara de un paciente xantoderma con cromosoma 18 en anillo y luego de comparar los resultados con las normas japonesas, encontraron una reducción en la longitud y anchura de la base del cráneo, crecimiento tardío de la mandíbula y todos los dientes con excepción del canino inferior, presentaban alteraciones de tamaño.

De todas las manifestaciones encontradas en este caso, la retención prolongada de dientes deciduos no ha sido relatada en casos anteriores, siendo esto además un hallazgo clínico importante presente en otros cuadros genéticos como el Síndrome de Carpenter o Acrocefalopolisindactilia Tipo II.

Un plan adecuado de prevención y un acompañamiento por un equipo multidisciplinario son necesarios en estos pacientes, ya que las alteraciones acontecen en todas las áreas ya sean sistémicas, físicas, emocionales o cognitivas. El paciente presentado recibió la atención odontológica necesaria y se encuentra bajo controles clínicos y radiográficos, esperando que sus condiciones de salud se normalicen para que se pueda iniciar la fase quirúrgica donde serán extraídos los dientes deciduos de retención prolongada.

\section{CONCLUSIONES}

El síndrome de cromosoma 18 en anillo es un trastorno poco común que necesita de más relatos y estudios que analicen sus condiciones y alteraciones, ya sean generales y/o bucales, para lograr una mayor comprensión de este fenómeno; proporcionando así, mayor información a los profesionales del equipo multidisciplinario, para que puedan promover mejorías en la calidad de vida de estos pacientes.

\section{Correspondencia:}

Rafael Celestino de Souza

Rua Doutor Bacelar, 1212. Vila Clementino,

São Paulo - SP, CEP 04026 - 000 Brasil.

Correo electrónico: rafacst@gmail.com

\section{REFERENCIAS BIBLIOGRAFICAS}

1. Christensen KR, Friedrich U, Jacobsen P, Jensen K, Nielsen J, Tsuboi T. Ring cromossoma 18 in mother and daugther. Res J Ment Defic. 1970; 14:49-67.

2. Thomas JV, Mezzasalma DF, Teixeira AM, et al. Growth hormone deficiency, hypothyroidism and ring chromosome 18: casereport.ArqBrasEndocrinol Metab. 2006; 50(5):951-956.

3. Dacou-Voutetakis C, Sertedaki A, Maniatis-Christidis $\mathrm{M}$, et al. Insulin dependent diabetes mellitus (IDDM) and autoimmune thyroiditis in a boy with a ring chromosome 18: additional evidence of autoimmunity or IDDM gene(s) on chromosome 18. J Med Genet. 1999; 36(2):156-8.

4. Fischer W, Dermitzel A, Osmers R, Pruggmayer M. Complete karyotype discrepancy between placental and fetal cells in a case of ring chromosome 18. Prenat Diagn. 2001; 21(6):481-3.

5. Dobos M, Fekete G, Raff R, et al. Ring chromosome 18: clinical, cytogenetic and molecular genetic studies on four patients. Int J Hum Genet. 2004; 4(3):197-200.

6. Kamiya TY, Rodrigues RM, Sandri RMCS, KokitsuNakata NM, Rodini ESO. Fístulas no lábio inferior em indivíduo comcromossomo 18 emanel. Anais do $54^{\circ}$ Congresso Brasileiro de Genética. 2008; 1(1):275.

7. Sripanidkulchai R, Suphakunpinyo C, Jetsrisuparb C, Luengwattanawanich $\mathrm{S}$. Thai girl with ring chromosome 18 (46XX, R18). J Med Assoc Thai. 2006; 89(6):878-81.

8. Koç A, Kan D, Karaer K, et al. An unexpected finding in a child with neurological problems: mosaic ring chromosome 18. Eur J Pediatr. 2008; 167(6):655-9.

9. Ono T, Okuma M, Hamada T, Motohashi N, Moriyama $\mathrm{K}$. A case of syndrome of ring chromosome 18 treated with a combined orthodontic-prosthetic approach. Cleft Palate Craniofac J. 2010; 47(2):201-10.

10. Bagherizadeh E, Behjati F, Saberi SH, Shafeghati Y. Prenatal diagnosis in a mentally retarded woman with mosaic ring chromosome18. Indian J Hum Genet. 2011; 17(2):111-3.

Recibido: $15 / 12 / 2013$

Aceptado: 07/01/2014 